\title{
Biomechanical Analysis of C5-C6 Spinal Unit with Prosthetic Disc by Finite Element Method
}

\author{
R. ÖZMEN ${ }^{a *}$, M. GÜNAY ${ }^{b}$ AND T. DEMIR ${ }^{c}$ \\ ${ }^{a}$ Karabuk University, Department of Mechatronics Engineering, Karabuk, Turkey \\ ${ }^{b}$ Karabuk University, Department of Mechanical Engineering, Karabuk, Turkey \\ ${ }^{c}$ TOBB Economy and Technology University, Department of Mechanical Engineering, Ankara, Turkey

\begin{abstract}
Spinal fusion and total disc arthroplasty are used in the surgical treatment of cervical disc degeneration. When mobility is desired in the functional spinal unit, total disc arthroplasty is preferred instead of fusion. In this research, the effects of artificial disc prosthesis on the biomechanical behaviour of the C5-C6 functional spinal unit were investigated via finite element method. Firstly, three-dimensional CAD models of C5 and C6 vertebrae were created by using a computerized tomography images of a healthy human neck via 3D Slicer computer software. The mobility of the healthy model consisting of intervertebral disc, joints, and soft tissues was validated by previously published experimental studies in the field. On the second step, a ball and socket type artificial disc was defined using connector elements between C5-6 functional spinal unit to simulate the total disc arthroplasty method. Finally, to investigate the effect of prosthesis core radius changes on the biomechanical properties of functional spinal unit, healthy and implanted models were analysed via finite element method.
\end{abstract}

DOI: 10.12693/APhysPolA.135.739

PACS/topics: finite element analysis, biomechanics, artificial disc, cervical spine, motion

\section{Introduction}

Intervertebral discs are fibrocartilage pads lying among the vertebra of the spine, which allows bending and twisting of the vertebral column, and distributes the loads to the adjacent vertebral bodies. Depending on the age progression, striking differences can occur in shape, volume, structure, and composition of the discs that may reduce motion capability with changing the mechanical characteristics of the spine $[1,2]$. In such extreme cases, the anterior cervical discectomy and fusion is accepted as a gold standard due to providing high fusion rates after implantation in the treatment of various cervical diseases. Following the long-term clinical investigations, it has been shown by various studies that cervical fusion is associated with adjacent segment degeneration. Total disc arthroplasty (TDA) is an alternative method to fusion due to the preservation of the mobility at the operated segment and considered to have the ability to prevent or at least slow down the early stage adjacent segment degeneration by reducing the abnormal stress distribution [3].

In the development process of a spinal implant, the effect of implant on the global kinetics and kinematics of the spine should be taken in account. Load sharing between the implant and the biological structure can also be investigated by the finite element (FE) model mimicking the physiological behavior of the spine or spinal motion segments [4]. In such studies, Galbusera et al. investigated the impact of single level disc arthroplasty on the biomechanics of C4-7 cervical spine segment by FE

*corresponding author; e-mail: ramazanozmen@karabuk.edu.tr method [5]. In the developed model, a nonlinear finite element model of the healthy C4-7 segment was investigated for flexion-extension motions. Afterwards, the Bryan disc prosthesis was built and positioned at C5-6 level. They concluded that the transmitted general forces through the facet joints, the segmental motion, and the physiological kinematics were preserved subsequently in the disc arthroplasty in terms of the healthy model.

This study is aimed to investigate the biomechanical behaviour of the cervical functional spin unit (FSU) implanted with a ball and socket type disc prosthesis according to the biomechanics of the healthy segment. For this reason, 3D FE model of the healthy C5-C6 spinal unit is constructed and verified by using experimental data based on cervical flexion-extension, axial rotation, and lateral bending studies. The simulation of the TDA method was performed with completely extracting the intervertebral disc and the anterior longitudinal ligaments from the FE model. The artificial disc is defined between endplates by connector elements at the specified neutral prosthesis position. The radius of the disc is assumed as 4,6 , and $8 \mathrm{~mm}$. Finally, a comparison is made between the simulations of healthy and implanted spinal units to determine the effects of prosthesis core radius on the biomechanics of the FSU.

\section{Material and methods}

A 3D model of the C5-C6 functional spinal unit was constructed for FE analysis depending on computerized images of a healthy cervical spine. The model includes bony sections (cortical, cancellous, and posterior), intervertebral disc with the sections of annulus, nucleus, and cortical end plates, facet joints, and five major ligaments. The cortical and cancellous bone sections of the verte- 
brae were defined separately and modelled using eight node solid elements. The intervertebral disc was partitioned into two sections as a central nucleus surrounded by the annulus. The fluid behavior of the nucleus was modelled via a nearly incompressible material. The superior and inferior surfaces of the disc were enclosed with a cartilaginous end-plate having an average thickness of $0.6 \mathrm{~mm}$. The complex structure of the annulus was defined by the Holzapfel-Gasser-Ogden (HGO) material formulation. The HGO model divides the Helmholtz free strain energy function $(\Psi)$ into different components corresponding to the ground substance, the fibers, and the material compressibility and given in the following form [4]:

$$
\begin{aligned}
& \Psi=C_{10}\left(\bar{I}_{1}-3\right)+\frac{1}{D}(J-1)^{2} \\
& +\frac{K_{1}}{2 K_{2}} \sum_{i=4,6}\left[\exp \left(K_{2}\left(\bar{I}_{i}-1\right)^{2}-1\right)\right]
\end{aligned}
$$

Here, $\bar{I}_{1}$ is the first deviatoric strain invariant of modified right Cauchy-Green tensor, $J$ is determinant of the deformation gradient, and the $\bar{I}_{i}(i=4,6)$ invariants are the square of the stretches in the fiber directions. The compressibility and stiffness of the ground substance are described by $D$ and $C_{10}$, respectively, $K_{1}>0$ and $K_{2}>0$ are material parameters. The annulus is divided into four parts as anterior inner (AI), anterior outer (AO), posterior inner $(\mathrm{PI})$ and posterior outer $(\mathrm{PO})$. The material properties for the sections of annulus is taken from Ref. [6]. Five major cervical ligaments consisting of anterior longitudinal, posterior longitudinal, capsular, ligamentum flavum, and interspinous ligaments were modeled with 1D truss elements acting only in tension nonlinearly. The hypoelastic material definition of ABAQUS was used to define ligament properties. The used material properties for defining the bony structures and ligaments were taken from Ref. [7]. For defining the facet joints the method of Kallemeyn et al. [8] was used. Rolhman et al. [9] stated that the modelling of the disc implant with kinematic coupling instead of conventional volumetric elements slightly alter the kinematics of the implanted segment. Therefore, a kinematic coupling is defined with connector elements to model the prosthesis. The core radius of the prosthesis is assumed as 4, 6 , and $8 \mathrm{~mm}$ in such a way that the rotation centres remained on the same line in the specified prosthesis position as described in Fig. 1b. The frictional effects of the rolling surfaces of the prosthesis were neglected. The developed FE model and the schematic representation of the prosthesis are given in Fig. 1.

The FE simulations of healthy C5-C6 functional spinal unit were performed in flexion and extension by applying only moment loads from 0 to $2.0 \mathrm{~N} \mathrm{~m}$. On the simulations of axial rotation and lateral bending, the moments were applied from 0 to $1.0 \mathrm{~N} \mathrm{~m}$. Moment loads were conducted to the model via defined surface on the superior aspect of the C5 vertebral body and the nodes on the inferior surface of C6 were constrained during the analysis.

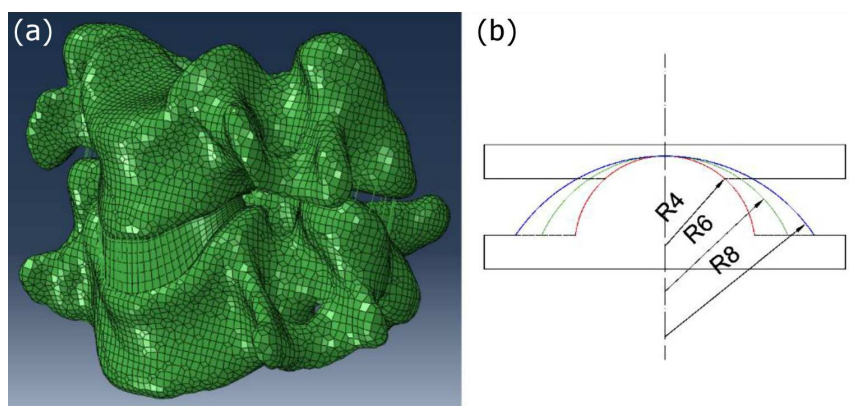

Fig. 1. (a) FE model of the C5-C6 FSU and (b) the rotation center definitions of the simulated artificial discs.

\section{Results and discussion}

The healthy C5-C6 model was analysed to assess the moment rotation behaviour under the flexion-extension, lateral bending, and axial rotation movements. In order to verify the finite element simulations, the comparison curves were taken from in vitro experimental measurements performed by several authors using similar loading and boundary conditions.

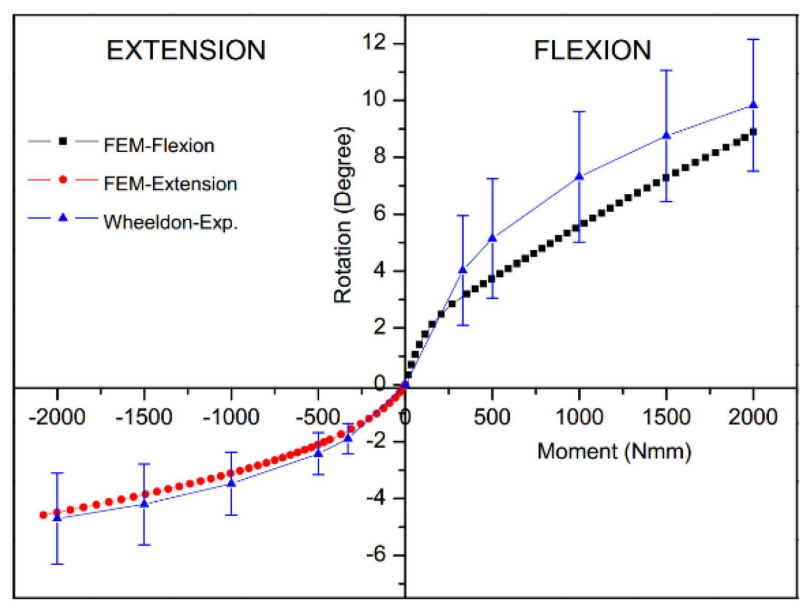

Fig. 2. Comparison of the extension-flexion motion for the C5-C6 healthy model.

The findings from the simulations of flexion-extension motion were compared with the experimental results of Wheeldon et al. [10], and the moment rotation graphs are given in Fig. 2. On the evaluation of moment rotation curves, the extension motion exhibited a little stiffness according to the flexion motion in agreement with the compared study. The predicted motion ranges of the healthy C5-C6 model for the flexion-extension simulations under only moment loading agreed reasonably by Ref. [10] (Fig. 2). For the extension motion, the closer results are obtained in the Wheeldon study. In the flexion motion the FE model showed slightly lower stiffness compared to the experimental study. However, the obtained moment rotation behavior remained within the standard deviation limits of the compared study. 
Verification of the axial rotation and lateral bending simulations were done by comparing the results with experimental corridors given by Yoganandan $[11,12]$. The moment rotation graphs of the healthy C5-C6 model obtained from the axial rotation and lateral bending simulations are given in Fig. 3a and b. The axial rotation motion range of the investigated spinal unit was obtained within the boundaries of the experimental corridor of Yoganandan [11]. On the other hand, the lateral bending motion ranges estimated by the FEM is stiffer than those determined by Yoganandan et al. [12].
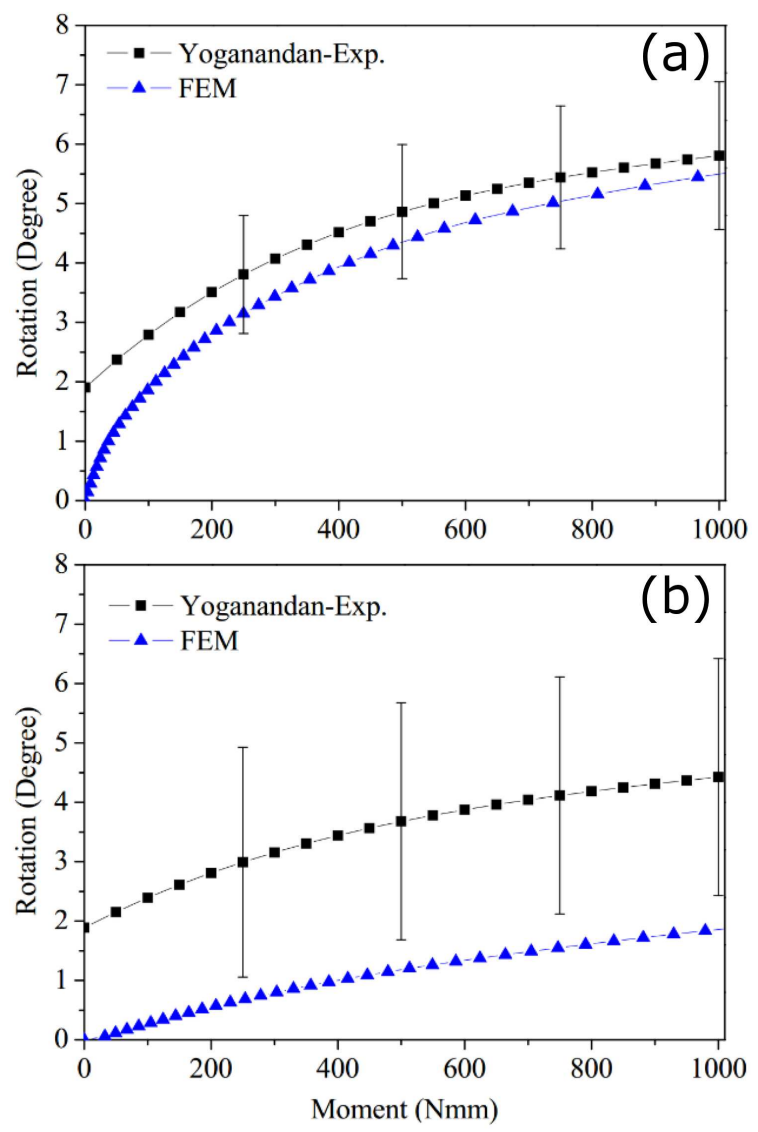

Fig. 3. Comparison of the (a) axial-rotation and (b) lateral bending motions for the C5-C6 healthy model.

FE method was used to study the effects of varying the core radius of the artificial disc prosthesis on the biomechanics of C5-C6 functional spinal unit. When the core radius of the prosthesis is changed, the rotation center is defined to remain on the same line during FEM simulations, as can be seen in Fig. 1. The range of motion (ROM) of the implanted C5-C6 spinal unit was compared with the healthy spine for the flexion-extension, axial rotation, and lateral bending motions. The comparison results between the two models are given in Fig. 4 .

The effects of the prosthesis on the ROM of the C5-C6 segment are lower than the healthy model in the flexion motion. However, during the flexion, the ROM of the C5-C6 level is decreased by reducing the core radius of the prosthesis. In the extension motion, it is observed

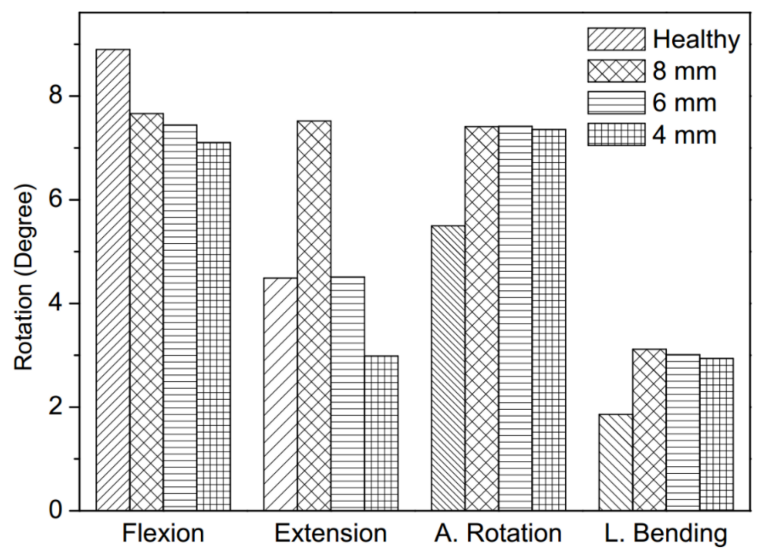

Fig. 4. ROM of the healthy and implanted models with different core radius.

that the ROM of the prosthesis with a radius of $8 \mathrm{~mm}$ is higher than the healthy model, and the prosthesis with a radius of $6 \mathrm{~mm}$ is approximately same as the healthy model. When the effect of the core radius of the prosthesis on the ROM of the implanted model was examined, it was seen that the ROM of the implanted model is reduced with decrease in the core radius of the prosthesis for both on the flexion and extension motions. In the axial rotation and lateral bending motions, it is observed that the ROMs of the prosthesis are higher than healthy model and the core radius variation has negligible effect on the ROM.

\section{Conclusion}

In this study, the biomechanical behavior of the C5C6 FSU implanted with a ball and socket type disc prosthesis is investigated and compared with the biomechanics of the healthy FSU. 3D model of the healthy C5-C6 FSU was constructed from CT scanned images with the help of 3D Slicer software. Verification of the healthy model is achieved with comparison of the biomechanical data given in the literature. An artificial disc prosthesis is defined between endplates of the C5-C6 vertebra after removing the intervertebral disc and anterior longitudinal ligament. Finally, the effects of prosthesis core radius on the biomechanics of the FSU were investigated by comparing to the healthy FSU. In flexion, the implanted model exhibited a stiffer behaviour compared to the healthy model. This drives lower ROM values achieved than the healthy model. On the evaluation of extension motion, higher and lower ROM values observed than healthy model for the core radius values of $8 \mathrm{~mm}$ and $4 \mathrm{~mm}$, respectively. However, as an overall behaviour, the ROM of the implanted model decreased as the core radius of the prosthesis decreased for both flexion and extension motion analysis. The higher range of motions observed in the implanted model than healthy model for the lateral bending and axial rotation investigations. A considerable variation was not observed in the ROM during the core radius alterations. 


\section{References}

[1] N. Newell, J.P. Little, A. Christou, M.A. Adams, C.J. Adam, S.D. Masouros, J. Mech. Behav. Biomed. Mater. 69, 420 (2017).

[2] J. Buckwalter, Spine (Phila. Pa. 1976) 20, 1307 (1995)

[3] P.A. Anderson, J.P. Rouleau, Spine (Phila. Pa. 1976) 29, 2779 (2004).

[4] R. Eberlein, G.A. Holzapfel, C.A.J. Schulze-Bauer, J. Comp. Meth. Biomech. Biomed. Eng. 4, 209 (2001).

[5] F. Galbusera, C.M. Bellini, M.T. Raimondi, M. Fornari, R. Assietti, Med. Eng. Phys. 30, 1127 (2008).

[6] M. Rao, Ph.D. Thesis, University of Denver, 2012.
[7] N.M. Shahraki, A. Fatemi, V.K. Goel, A. Agarwal, Front. Bioeng. Biotechnol. 3, 1 (2015).

[8] N.A. Kallemeyn, S.C. Tadepalli, K.H. Shivanna, N.M. Grosland, Comp. Meth. Prog. Biomed. 95, $227(2009$.

[9] A. Rohlmann, T. Zander, B. Bock, G. Bergmann, Proc. Inst. Mech. Eng. H J. Eng. Med. 222, 229 (2008).

[10] J.A. Wheeldon, F.A. Pintar, S. Knowles, N. Yoganandan, J. Biomech. 39, 375 (2006).

[11] N. Yoganandan, B.D. Stemper, F.A. Pintar, J.L. Baisden, B.S. Shender, G. Paskoff, Spine (Phila. Pa. 1976) 33, 490 (2008).

[12] N. Yoganandan, F.A. Pintar, B.D. Stemper, C.E. Wolfla, B.S. Shender, G. Paskoff, J. Bone Jt. Surg. Ser. A 89, 1066 (2007). 